\title{
Hepatoprotective Effects of Polysaccharide from Anoectochilus roxburghii (Wall.) Lindl. on Rat Liver Injury Induced by $\mathrm{CCl}_{4}$
}

\author{
Xiaoling $\mathrm{Yu}^{1,2}$ \\ Lingyi Huang $\left.\mathbb{D}^{\prime}\right)^{\prime}$ \\ Chen You $\mathbb{1 D}^{2}$ \\ Liying Huang'
}

'School of Pharmacy, Fujian Medical University, Fuzhou, Fujian, People's Republic of China; ${ }^{2}$ Department of Pharmacy, Mengchao Hepatobiliary Hospital of Fujian Medical University, Fuzhou, People's Republic of China

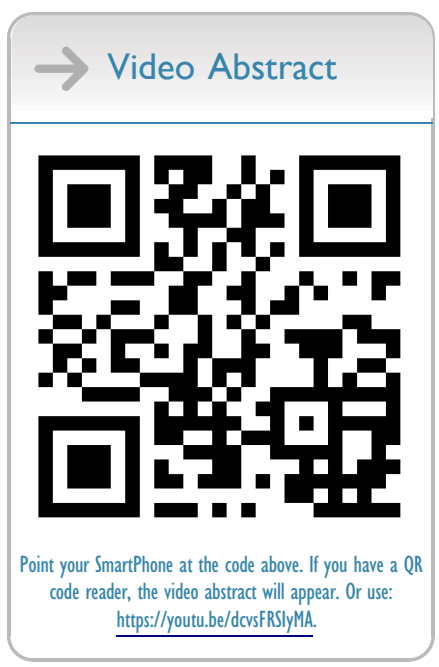

Correspondence: Liying Huang School of Pharmacy, Fujian Medical University, No.I Xuefu North Road University Town, Fuzhou, Fujian, 350122, People's Republic of China

Tel +86 I3860635I39

Fax +8659122862016

Email fjmuhly88@sina.com.cn
Purpose: The polysaccharide of Anoectochilus roxburghii (wall.) Lindl. (ARPS) is one of its important active ingredients. Hepatoprotective effects of ARPS on rat liver injury induced by $\mathrm{CCl}_{4}$ were studied.

Methods: ARPS was extracted using the ultrasonic method and successfully purified by high-speed counter-current chromatography (HSCCC) with a two-phase aqueous system composed of $12.5 \%$ PEG $1000-20 \% \mathrm{~K}_{2} \mathrm{HPO}_{4}: \mathrm{KH}_{2} \mathrm{PO}_{4}(1: 1)$. The HSCCC conditions were optimized, and the structure of ARPS was characterized. The hepatoprotective effects of ARPS against $\mathrm{CCl}_{4}$-induced chronic hepatic injury in SD rats were evaluated.

Results: The results showed that ARPS was a water-soluble polysaccharide with a molecular weight of 28,518 Da. It was composed of mannose, ribose, glucose, and arabian sugar; its monosaccharide molar ratio was glucose:ribose:arabinose:mannose = 54.24:13.20:1.09:1.00. The purity of ARPS was determined by HPLC to be $96.93 \%$. The intervention effects of ARPS on $\mathrm{CCl}_{4}$-induced hepatic damage model in rats showed that ARPS could effectively reduce the activity of alanine amino transferase and aspartate amino transferase, decrease the content of malondialdehyde and nitric oxide synthesis, and increase the content of glutathione. Pathology revealed that liver plate order, liver cell degeneration, and edema were improved; inflammatory cell infiltration was not observed after ARPS intervention.

Conclusion: ARPS had the function of antioxidant for protecting $\mathrm{CCl}_{4}$-induced injured liver, and the mechanisms were related to anti-lipid peroxidation, which could eliminate oxygen-free radicals and protect liver cells from attacks by free radicals.

Keywords: Anoectochilus roxburghi, polysaccharide, $\mathrm{CCl}_{4}$-induced hepatic damage, hepatoprotective effects, rat

\section{Introduction}

Anoectochilus roxburghii (Wall.) Lindl. (A. roxburghii), also known as Jin Xian Lian in Chinese and "the king of medicine", 1,2 is widely distributed in Southern China, ${ }^{3}$ especially in Fujian and Guangdong. ${ }^{4}$ In recent decades, many studies have shown that $A$. roxburghii, as a Chinese folk medicine, has diverse pharmacological effects and medicinal properties, such as hepatoprotection, ${ }^{5-9}$ anti-diabetes, ${ }^{10,11}$ antifatigue, ${ }^{12}$ antiosteoporosis,,${ }^{9,13,14}$ vascular protective, ${ }^{15}$ antioxidation, ${ }^{16,17}$ and antitumor. $^{18,19}$ Some scholars have applied advanced and efficient technologies in the extraction, separation, and structural characterization of the active ingredients from $A$. roxburghii because of its curative effects. In recent years, the research team 
of the authors has conducted an in-depth exploration of the active components of $A$. roxburghii. The results showed that the components contained alkaloid, ${ }^{20}$ nucleosides, ${ }^{21}$ nucleotide, ${ }^{21}$ sterol, $^{22}$ triterpenoids, ${ }^{23}$ flavonoid, ${ }^{24,25}$ and gastrodine. ${ }^{26}$ In addition, the pharmacological effects and mechanism of $A$. roxburghii were also studied. ${ }^{10,27,28}$ For example, the polysaccharide from artificially cultivated A. roxburghii has been reported to possess antitumor effects. $^{19}$

However, little information has been published in terms of purification and structural characterization of the polysaccharide from $A$. roxburghii and its effects on $\mathrm{CCl}_{4}$ induced liver injury by in rats thus far. Some reports are available on the hepatoprotective effects of $A$. roxburghii from the different genera of the same family. For instance, Shih et al confirmed that the aqueous extract from Anoectochilus formosanus could significantly improve the liver fibrosis caused by dimethylnitrosamine in mice. $^{29} \mathrm{Wu}$ et al reported that the aqueous extract from $A$. formosanus could improve the hepatic fibrosis induced by thioacetamide and inhibit the activity of Kupffer cells. ${ }^{30}$ Furthermore, a study by Yang et al revealed that preparation of ARPT from $A$. roxburghii has antiinflammatory, antioxidative, and antiapoptotic effects on hepatic injury in carbon tetrachloride $\left(\mathrm{CCl}_{4}\right)$-induced mice. $^{8}$

The present study aimed to find the best method to separate polysaccharides from $A$. roxburghii by using high-speed counter-current chromatography (HSCCC) and preliminarily identify their structure, which is the fundamental research direction for the further investigation of their pharmacological effects and mechanism. The structure of ARPS was characterized, and it confirmed that ARPS was a water-soluble polysaccharide with increased purity. However, to the knowledge of the authors, little information is known about the hepatoprotective activities of such polysaccharides on $\mathrm{CCl}_{4}$-induced liver injury. Therefore, this study also aimed to investigate the hepatoprotective activities and possible mechanisms underlying the hepatoprotective effects of polysaccharides from $A$. roxburghii.

\section{Materials and Methods}

\section{Reagents and Materials}

Anoectochilus roxburghii (Wall.) Lindl. (A. roxburghii), also known as Jin Xian Lian in Chinese, was purchased from Fujian Wuyishan Luzhou Group (Fujian, China) and identified by Professor Zhang Yonghong (Department of Pharmaceutical Chemistry, Fujian Medical University). A. roxburghii is planted in Moso bamboo forest. It was cultivated from February to September under the conditions of $800 \mathrm{~m}$ altitude, approximately $80 \%$ shade rate, and $75 \%$ relative humidity. Dialysis tubing MD44 with a molecular weight cutoff of 8000-14,000 Da was purchased from Beijing Solaibao Technology Co., Ltd. (Beijing, China). All standard substances were supplied by Sigma-Aldrich Reagent Co., Ltd. (St. Louis, MO, USA). Methanol and acetonitrile of chromatographic grade were provided by Merck (Merck KGaA, Germany). SD rats (SPF grade) were obtained from the Experimental Animal Centre of Chinese Academy of sciences (Shanghai, China. SCXK 2012-0002). Reagent kits for measuring alanine amino transferase (ALT), aspartate amino transferase (AST), glutathione (GSH), nitric oxide synthesis (NOS) and malondialdehyde (MDA) were purchased from Nanjing Jiancheng Institute of Bioengineering and Technology (Nanjing, China). Mannose, ribose, rhamnose, glucose, arabinose, xylose, and galacturonic acid were obtained from Sigma Chemical Co (St. Louis, USA). All other chemical reagents were of analytical grade and obtained from Sinopharm Chemical Reagent Co., Ltd (Shanghai, China). Distilled water was acquired from a Milli-Q purification system (Millipore, USA). This study was approved by the Institutional Ethical Committee of the Faculty of Medicine, Mengchao Hepatobiliary Hospital of Fujian Medical University, Fujian, China (No. 2020_051_01).

\section{Instruments}

The HSCCC (TBE-300A, Shanghai Tauto Biological Company, China) was equipped with a temperature and rotary-speed controlling system, a BSZ-100 automatic fraction collector (Shanghai Huxi Analytical Instrument Factory), a SpectraMax M5 (Molecular Devices, USA), an LC-1100 high-performance liquid chromatograph (Agilent, USA), a Nicolet iS50 FTIR spectrometer (ThermoFisher Scientific Inc., USA), and an LC-1260 HPLC instrument (Agilent, USA).

\section{Extraction of Polysaccharides}

\section{A. roxburghii Preprocessing}

The harvested $A$. roxburghii required pretreatment. It was washed cleanly with water and then dried below $60{ }^{\circ} \mathrm{C}$ until reaching a constant weight. Afterwards, the fresh 
A. roxburghii became dried herbs, which were smashed into dried homogenized powder that was screened through a mesh size of 80 with a high-speed disintegrator.

\section{Extraction of Crude Polysaccharide}

The dried powder of $A$. roxburghii (100 g) was extracted three times with distilled water $(2 \mathrm{~L})$ at $60{ }^{\circ} \mathrm{C}$ for $30 \mathrm{~min}$ under ultrasonic wave. After centrifugation at $4200 \mathrm{rpm}$ for $10 \mathrm{~min}$, the supernatant was collected and concentrated. The concentrated solution was deproteinized by Sevag method (chloroform: n-butanol $=4: 1$ ). The mixtures were shocked three times at $250 \mathrm{rpm}$ for $20 \mathrm{~min}$ and then centrifuged at $4200 \mathrm{rpm}$ for $10 \mathrm{~min}$. Afterwards, metaprotein and organic solvents were discarded, and the supernatants were reserved and precipitated by anhydrous ethanol at a ratio of $1: 4$ under $4{ }^{\circ} \mathrm{C}$ overnight. Finally, after centrifugation at $4200 \mathrm{rpm}$ was performed for $10 \mathrm{~min}$, the precipitates were collected using the evaporator at $30{ }^{\circ} \mathrm{C}$ under the vacuum condition to drive away the ethanol and then dried at $-20{ }^{\circ} \mathrm{C}$ using a vacuum-freezing dryer until constant weight and dried powders were achieved; these powders were the crude polysaccharide of $A$. roxburghii (CARPS, $16 \mathrm{~g}$ ).

\section{Purification of CARPS}

\section{Selection of Aqueous Biphasic System of HSCCC}

In accordance with the recommendation and rules of twophase solvent system, ${ }^{31}$ the influences of PEG with different molecular weights $(600,800$, and $1000 \mathrm{Da})$ and phosphates $\left(\mathrm{K}_{2} \mathrm{HPO}_{4}\right.$ and $\left.\mathrm{KH}_{2} \mathrm{PO}_{4}\right)$ with different proportions of the partition coefficient $(K)$ were studied. Phosphates were thoroughly dissolved in distilled water and then equally mixed together with dissolved PEG. After equilibrium was achieved, the miscible mixtures turned into supernatant liquid as the upper layer (the stationary phase) and a subnatant liquid as the lower layer (the mobile phase), whose volumes were recorded. CARPS ( $5 \mathrm{mg}$ ) was added to the two mutually equilibrated solvent phases ( $2 \mathrm{~mL}$ each) in a stoppered test tube and completely mixed with the vortex to balance the contents. After stratification, an equal volume of the upper and lower layers $(500 \mu \mathrm{L})$ each was transferred into a separate test tube. Then, the polysaccharide concentration of CARPS in both phases was measured using a spectrophotometer at an appropriate wavelength to obtain the $A_{U}$ and $A_{L}$ values via phenol-sulfuric acid method. $K$ was expressed as follows:

$$
K=\frac{A_{u}}{A_{L}}
$$

where $A_{\mathrm{u}}$ and $A_{\mathrm{L}}$ are the absorbance of polysaccharide by the phenol-sulfuric acid method in the upper and lower layers, respectively.

Meanwhile, $2 \mathrm{~mL}$ of each phase was transferred into a test tube (total volume of $4 \mathrm{~mL}$ ). This tube was plugged and inverted several times and then immediately placed in an upright position to determine the layered time called for the two phases to become a distinct interface.

\section{Flow Rate of Mobile Phase and Revolution Speed Effect on Retention Ratio of Stationary Phase}

The effect of different flows of the mobile phase and different revolution speeds on the stationary phase was explored. First, the column of HSCCC was entirely filled with the stationary phase until its flow from the column outlet to ensure that no air was present in the column. Then, by testing various revolution speeds, the mobile phase was pumped into the column at different flow rates. Finally, the mobile phase constantly flowed from the column outlet until the two-phase solvent system reached hydrodynamic equilibrium in the column of HSCCC where the stationary phase stopped to flow. During the whole process, the stationary phase flowing out of the column was collected in a measuring cylinder, and its volume was measured. The retention ratio of the stationary phase $(R)$ was calculated as follows: ${ }^{31}$

$$
R(\%)=\frac{V_{t}-V_{f}}{V_{t}} \times 100 \%
$$

where $V_{\mathrm{t}}$ is the total volume pumped into the column of the stationary phase, and $V_{\mathrm{f}}$ is the volume flowing from the column.

\section{Separation and Purification of CARPS by HSCCC}

As mentioned, a two-phase solvent system reached hydrodynamic equilibrium in the column of HSCCC at a selected flow rate of the mobile phase and desired revolution speed. Then, the CARPS (200 mg) dissolved in the mobile phase $(20 \mathrm{~mL})$ was injected into the column. Afterwards, the effluents from the outlet of the column were continuously collected $(5 \mathrm{~mL}$, each tube, every 5 min), and their color change was monitored using the phenol-sulfuric acid method.

After fractions were collected, the effluents belonging to the same polysaccharide component were combined and evaporated into concentrated solutions in a vacuum. The concentrated solutions were dialyzed with a dialysis bag, 
which cut off $8000-14,400$ molecular weight (Mw) to remove phosphate. After small molecular substances, especially phosphate, were removed completely, all solutions were freeze-dried at $-20{ }^{\circ} \mathrm{C}$ in a vacuum freeze dryer until their weight was constant to that of dried powder, which was the purified ARPS, and then stored in vacuum dryer at normal temperature for subsequent use.

\section{Characterization of ARPS}

\section{Analysis of Homogeneity and Determination of} Average Molecular Weight

The ARPS $(2 \mathrm{mg})$ dissolved in distilled water $(5 \mathrm{~mL})$ was detected with an ultraviolet and visible spectrophotometer and then scanned in the range of 200-800 nm. ARPS $(5 \mathrm{mg})$ was dissolved in distilled water $(1 \mathrm{~mL})$, analyzed via high-performance gel-permeation chromatography on an LC instrument equipped with the column of tiger kin ODS $(5 \mu \mathrm{m}, 10 \times 250 \mathrm{~mm})$, and eluted with $0.7 \% \mathrm{Na}_{2} \mathrm{SO}_{4}$ solutions $^{32}$ at a flow rate of $1.0 \mathrm{~mL} / \mathrm{min}$ through monitoring via refractive index detector. A series of known molecular mass Dextrans (MW of 150, 80, 50, 12, and $5 \mathrm{KDa}$ ) were used for standard substances, and a calibration curve was created to calculate the molecular weight of ARPS following the literature reported by Alosp and Vlachogiannis. $^{33}$

\section{Infrared Spectra Analysis}

Spectrum Two FTIR was used to detect the functional groups of ARPS (2 mg), which was evenly mixed with moderate $\mathrm{KBr}$ and pressed into slices. The mixture was then analyzed and scanned in the range of $400-4000 \mathrm{~cm}^{-1}$.

\section{Determination of Composition}

LC analysis was applied for quantification and identification of ARPS. The monosaccharide derivatization of 1-phenyl-3-methyl-5-pyrazolone (PMP) was implemented. ARPS $(3 \mathrm{mg}$ ) was hydrolyzed with $0.02 \mathrm{mmol} / \mathrm{L}$ of trifluoroacetic acid at $100{ }^{\circ} \mathrm{C}$ for $12 \mathrm{~h}$ and then cooled. After centrifugation at $8000 \mathrm{rpm}$ was conducted for $10 \mathrm{~min}$, the $\mathrm{pH}$ of supernatants was mediated by adding $0.1 \mathrm{~mol} / \mathrm{L}$ of $\mathrm{NaOH}$ as hydrolyzed sample solutions. Then, $0.5 \mathrm{~mol} / \mathrm{L}$ $100 \mu \mathrm{L}$ PMP and $0.1 \mathrm{~mol} / \mathrm{L}$ of $30 \mu \mathrm{L} \mathrm{NaOH}$ were added into the hydrolyzed samples of ARPS and various standard monosaccharides (mannose, ribose, rhamnose, glucose, arabinose, xylose, and galacturonic acid). The mixture was mixed and reacted in a bath at $70{ }^{\circ} \mathrm{C}$. Through the above procedure of hydrolysis and derivatization, the reaction solution was extracted with chloroform to remove PMP, and then the aqueous layer was filtered through a $0.45 \mu \mathrm{m}$ membrane for HPLC analysis. This analysis was performed using an HPLC instrument equipped with RP-C18 column $(4.6 \times 250 \mathrm{~mm}, 5 \mu \mathrm{m}$, Waters $)$ at a wavelength of $254 \mathrm{~nm}$. The eluotropic procedure was as follows: the concentrations of acetonitrile were $7 \%$, $19 \%, 25 \%$, and $45 \%$; the other solvent system was distilled water; the time changes were $0,4,13$, and $28 \mathrm{~min}$; and the flow rate was $0.8 \mathrm{~mL} / \mathrm{min}$.

\section{Hepatoprotective Effects of ARPS on $\mathrm{CCl}_{4}$-Induced Liver Injury in Rats}

The hepatoprotective effects of ARPS against $\mathrm{CCl}_{4}$ induced chronic hepatic injury in rats were evaluated. Specific pathogen-free grade SD rats (male, 6 weeks old) with body weights (BW) of $250 \pm 10 \mathrm{~g}$ were kept in standard conditions with controlled temperature $\left(23{ }^{\circ} \mathrm{C} \pm\right.$ $\left.1{ }^{\circ} \mathrm{C}\right)$ and humidity $(55 \% \pm 10 \%)$ under a 12 -h light/dark cycle and with free access to a standard laboratory pellet diet and water. The procedures involving animals strictly complied with Chinese law, specifically the use and care of laboratory animals.

After 1 week of adjustment, the rats were randomly divided into five groups (three for each group) in accordance with the preliminarily experimental results: (I) normal control group (NC group), (II) model control group (MC group), (III) low-dose group of ARPS $(100 \mathrm{mg} / \mathrm{kg} / \mathrm{d}$, LD group), (IV) medium-dose group of ARPS (200 mg/ $\mathrm{kg} / \mathrm{d}$, MD group), and (V) high-dose group of ARPS (400 $\mathrm{mg} / \mathrm{kg} / \mathrm{d}, \mathrm{HD}$ group). All rats except those in the $\mathrm{NC}$ group were administered with intraperitoneal injection of $\mathrm{CCl}_{4}(50 \%, 1 \mathrm{~mL} / \mathrm{kg}, \mathrm{BW})$ for 10 weeks until $\mathrm{CCl}_{4^{-}}$ induced liver injury models were successfully achieved. Then, the rats were administered with corresponding doses of ARPS. Blank blood samples were collected before administration, and then $4,8,12,24$, and $48 \mathrm{~h}$ were set for taking blood spots. Cell Counting Kit-8 (CCK-8) experiment was carried out to determine the dose of ARPS on $\mathrm{CCl}_{4}$-induced liver injury in rats and the time of blood sampling.

Meanwhile, the rats were randomly divided into four groups (five for each group): (I) normal control group (NC group), (II) $\mathrm{CCl}_{4}$-induced model control group (MC group), (III) lentinan treatment group (positive control group, PC group), and (IV) ARPS treatment group (ARPS group). All rats except those in the $\mathrm{NC}$ group were administered with intraperitoneal injection of $\mathrm{CCl}_{4}$ $(50 \%, 1 \mathrm{~mL} / \mathrm{kg}$, biw) for 10 weeks, while the $\mathrm{NC}$ group received physiological saline. After $\mathrm{CCl}_{4}$-induced liver 
injury models were successfully established, the rats in the ARPS group underwent ARPS treatment; the dose depended on the screening of CCK-8 experiment, while those in the PC group received lentinan $(200 \mathrm{mg} / \mathrm{kg}$, dissolved in physiological saline). All treatments were administered via intragastric gavage, once a day for 14 days in each group. After the last experimental medication was administered (at $24 \mathrm{~h}$ ), all rats were weighed. Blood samples were collected by retro-orbital bleeding, and the rats were sacrificed via cervical dislocation. The blood samples were separated into serum at $4{ }^{\circ} \mathrm{C}$. The activities of serum ALT and AST were determined using commercial reagent kits in accordance with the manufacturer's instructions via dinitrophenyl-hydrazine method. The levels of NOS and MDA were estimated according to the manufacturers' instructions by Thiobarbituric Acid Reactive Substance (TBARS) and spectrophotometry using kits obtained from Nanjing Jiancheng Institute of Bioengineering and Technology. The activities of GSH were measured using GSH Quantification kits acquired from Nanjing Jiancheng Institute of Bioengineering and Technology according to the manufacturers' instructions. The hepar from rats in the different groups was excised and fixed in $10 \%$ neutral formalin solution once, dehydrated in graded alcohol, and embedded in paraffin. Slices with a thickness of 4-5 $\mu \mathrm{m}$ were cut and stained with hematoxylin and eosin (H\&E) for photomicroscopic observation.

\section{Results}

\section{Optimization of Aqueous Biphasic System}

The aqueous biphasic system composed of PEG- $\mathrm{K}_{2} \mathrm{HPO}_{4-}$ $\mathrm{KH}_{2} \mathrm{PO}_{4}-\mathrm{H}_{2} \mathrm{O}^{34,35}$ with different molecular weights and proportions for HSCCC was introduced in the present study to obtain the optimum $K$ values. In accordance with the experiment, the proportion of PEG with different molecular weights $(600,800$, and 1000$)$ exceeded $12.5 \%$, while the solubility of ARPS in the upper phase decreased as the $K$ values declined. Meanwhile, the viscosity of the solution gradually increased with the increase in the amount of polymer, and then the layer time of the twophase solvent system was gradually prolonged. On the contrary, the proportion below 12.5\% PEG was difficult for the two-phase solvent system. Therefore, a proportion of $12.5 \%$ PEG was chosen to further optimize the aqueous biphasic system.

The effect of different molecular weights of $12.5 \%$ PEG and mass fractions of $\mathrm{K}_{2} \mathrm{HPO}_{4}-\mathrm{KH}_{2} \mathrm{PO}_{4}(1: 1)$ on $K$ and layer time was surveyed. When the mass fractions of $\mathrm{K}_{2}$ $\mathrm{HPO}_{4}-\mathrm{KH}_{2} \mathrm{PO}_{4}$ in PEG 600 and 800 were below 16\% and $18 \%$, respectively, the two-phase solvent system was difficult to separate. At PEG 1000 and $20 \%$ mass fraction of $\mathrm{K}_{2}$ $\mathrm{HPO}_{4}-\mathrm{KH}_{2} \mathrm{PO}_{4}(1: 1)$, the $K$ value was optimum and the layer time was the shortest. Therefore, PEG $1000-\mathrm{K}_{2} \mathrm{HPO}_{4}-$ $\mathrm{KH}_{2} \mathrm{PO}_{4}-\mathrm{H}_{2} \mathrm{O}(1.25: 1: 1: 6.75$, w/w) was the optimum aqueous biphasic system for purifying CARPS by HSCCC, of which the $K$ value was 0.868 and the layer time was $20 \mathrm{~s}$.

\section{Retention of Stationary Phase}

Successful separation in HSCCC widely relies on the ratio of the stationary phase reserved in the column. The higher the retention of the stationary phase, the better the resolution of the peak. ${ }^{36}$ Besides the separating time of the twophase solvent system, the optimum flow rate of the mobile phase and the suitable revolution speed of HSCCC apparatus considerably influence the retention ratio of the stationary phase in the column.

In this study, to protect the rotated gear composed of polytetrafluoroethylene materials in the HSCCC instrument, the temperature of the circulating water was set to $25^{\circ} \mathrm{C}$ to cool the large amount of heat caused by the high rotational speed. The flow rate of the stationary phase was $15 \mathrm{~mL} / \mathrm{min}$, which could reduce the waiting time. The following effects on the retention of the stationary phase were studied: flow rate of the mobile phase $(0.5,1.0,1.5$, $2.0,2.5$, and $3.0 \mathrm{~mL} / \mathrm{min})$ and revolution speed $(700,750$, $800,850$, and $900 \mathrm{rpm})$. As a result, the lower flow rate generally generated a higher retention ratio of the stationary phase and improved the peak resolution but required a longer separation time than the higher flow rate (as shown in Figure 1A). The higher the revolution speed, the higher the retention ratio of the stationary phase (Figure 1B). The high revolution speed simultaneously caused the immoderate solute band to broaden because of severe vibration under high pressure and too much heat to damage the instrument. However, the lower speed reduced the volume of the stationary phase reserved in the column and led to lowered peak resolution. In conclusion, the flow rate for the mobile phase was $1.0 \mathrm{~mL} / \mathrm{min}$, the flow rate for the stationary phase was $15.0 \mathrm{~mL} / \mathrm{min}$, and the revolution speed was $850 \mathrm{rpm}$, with $25{ }^{\circ} \mathrm{C}$ as the temperature of circulating water. These parameters were the optimal for the best retention ratio of the stationary phase $(32.59 \%)$. Satisfactory separation could still be obtained at a high $K$ value, although retention of over $50 \%$ is perfect. $^{36}$ 

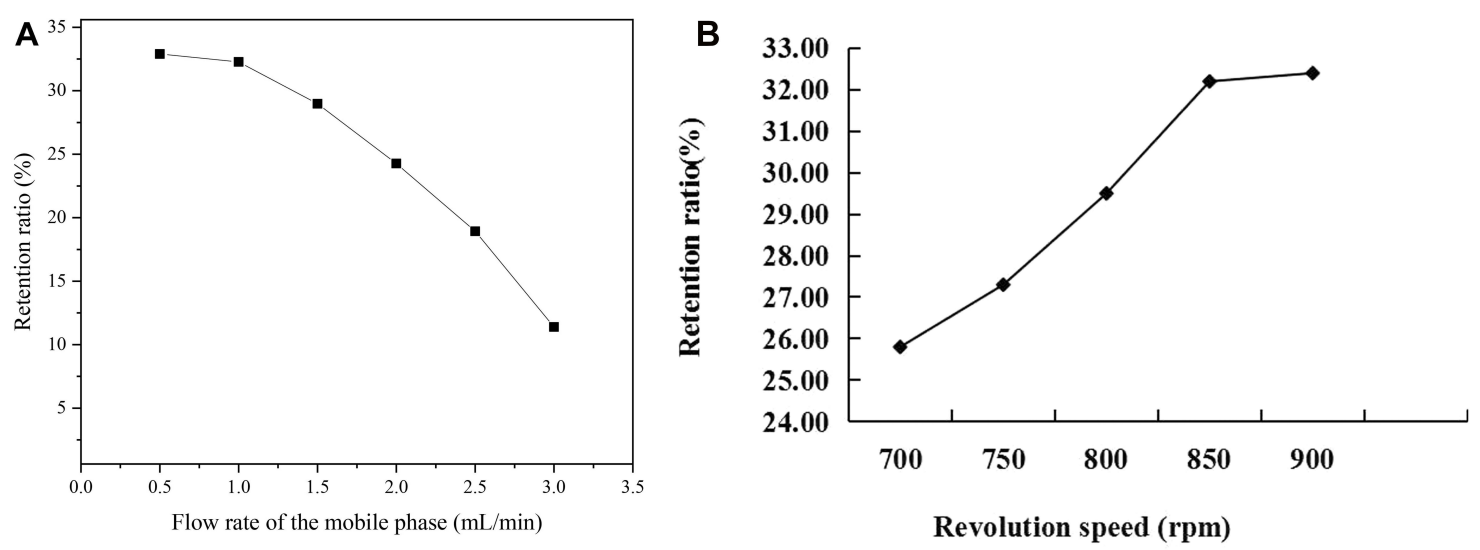

Figure I Effect of the flow rate of the mobile phase $(\mathbf{A})$ and the revolution speed $(\mathbf{B})$ on the retention of the stationary phase.

\section{Separation CARPS by HSCCC}

In accordance with the above experimental parameters, the aqueous biphasic system consisted of PEG $1000-\mathrm{K}_{2} \mathrm{HPO}_{4^{-}}$ $\mathrm{KH}_{2} \mathrm{PO}_{4}-\mathrm{H}_{2} \mathrm{O}(1.25: 1: 1: 6.75$, w/w), and separation was performed at a flow rate of $1.0 \mathrm{~mL} / \mathrm{min}$ for the mobile phase and $15.0 \mathrm{~mL} / \mathrm{min}$ for the stationary phase, with a revolution speed of $850 \mathrm{rpm}$ and $25{ }^{\circ} \mathrm{C}$ as the temperature of circulating water. The effluents were monitored at $482 \mathrm{~nm}$ using phenol-sulfuric acid method. The effluents from the outlet of the column were continuously collected ( $5 \mathrm{~mL}$, each tube, every $5 \mathrm{~min}$ ), and the fractions (37-57 tubes) that belonged to the same polysaccharide components were combined and monitored at $482 \mathrm{~nm}$ using the same method, as depicted in Figure 2. On the

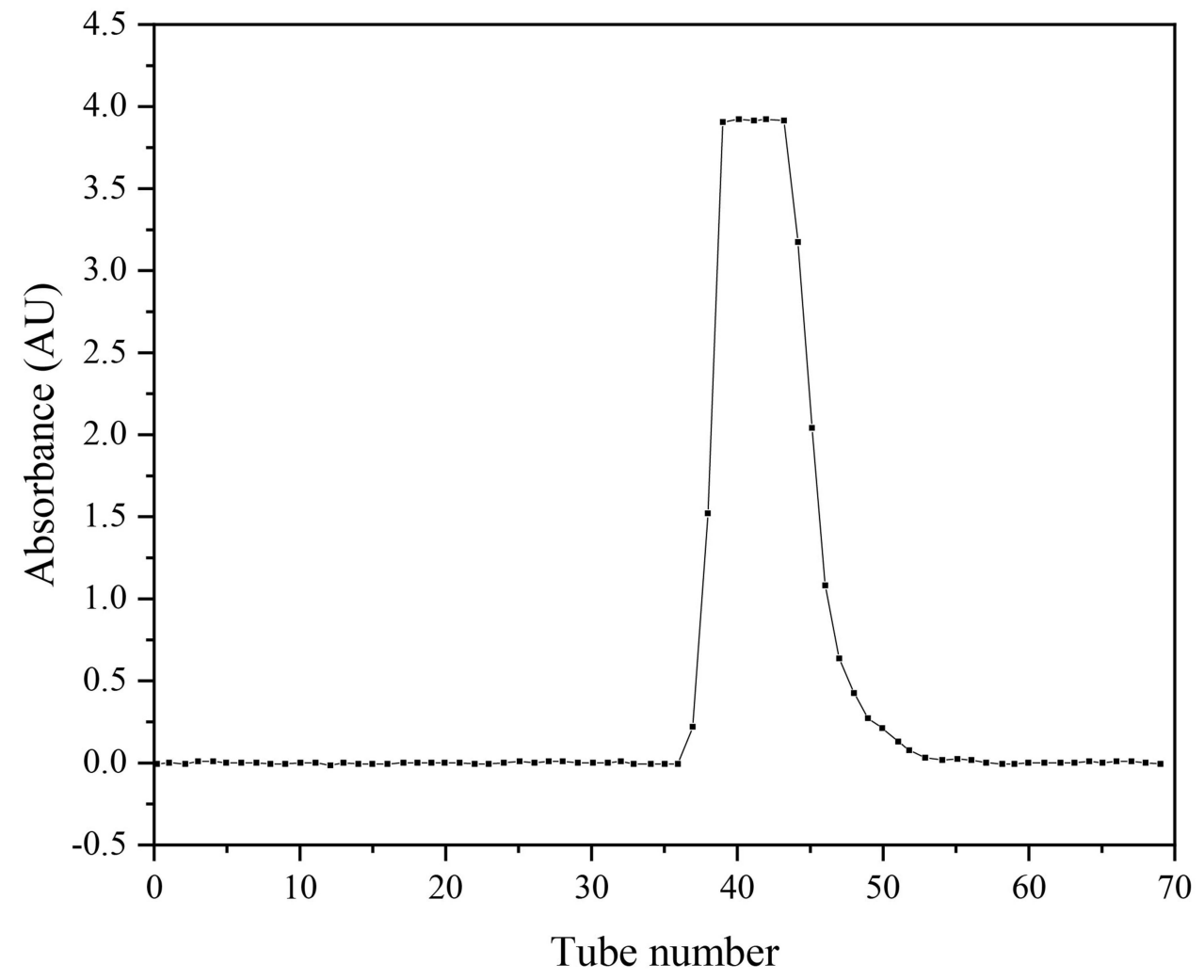

Figure 2 Spectrogram of ARPS collected via HSCCC and detected using phenol-sulfuric acid. 
basis of this method, the ARPS contained $96.93 \%$ carbohydrate, while the ARPS in CARPS was $67.09 \%$.

\section{Characterization of ARPS}

As indicated in Figure 3A, the ARPS purified by HSCCC had no absorption at 260 and $280 \mathrm{~nm}$ and no chemical reaction with Folin-phenol reagent, thus revealing the free protein and nucleic acid in ARPS.

The molecular weight of polysaccharides is usually determined using high-performance gel -permeation chromatography (HPGPC). ${ }^{37}$ The formula of the calibration curve correlating the molecular weight of ARPS was $\operatorname{lgM}_{\mathrm{W}}=-0.2382 \mathrm{t}+6.5932, \mathrm{R}^{2}=0.9902$, where $\mathrm{M}_{\mathrm{W}}$ stands for the average molecular weight of ARPS. HPGPC analysis of ARPS displayed a symmetrical and single sharp peak (Figure 3B), whose retention time was $11.959 \mathrm{~min}$. Its molecular weight was calculated to be 28,518 Da.
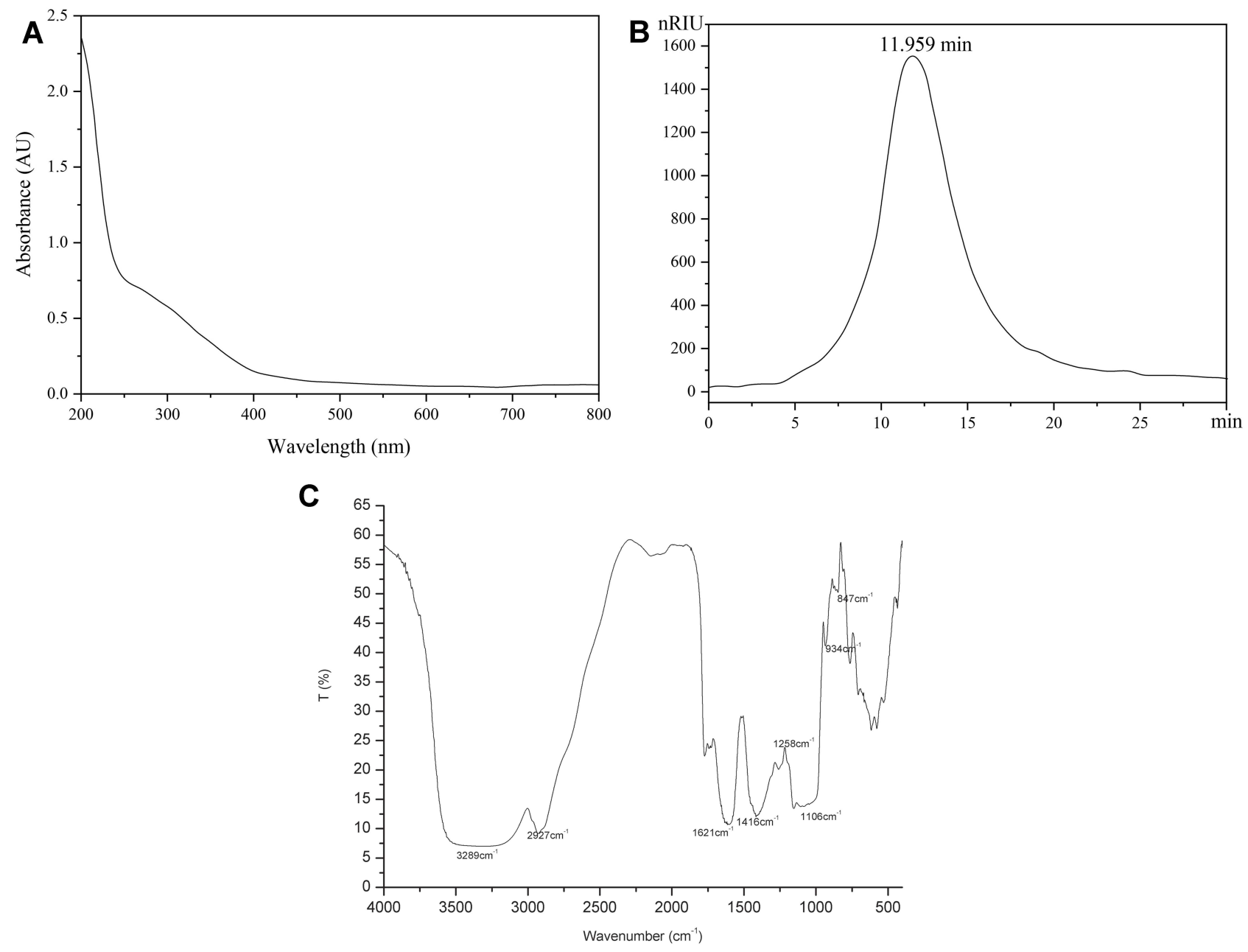

Figure 3 Characterization of ARPS. (A) UV-visible spectrum of ARPS in the range of 200-800 nm; (B) Profile of ARPS in HPGPC; (C) FT-IR spectrum of ARPS in the range of $400-4000 \mathrm{~cm}^{-1}$. 

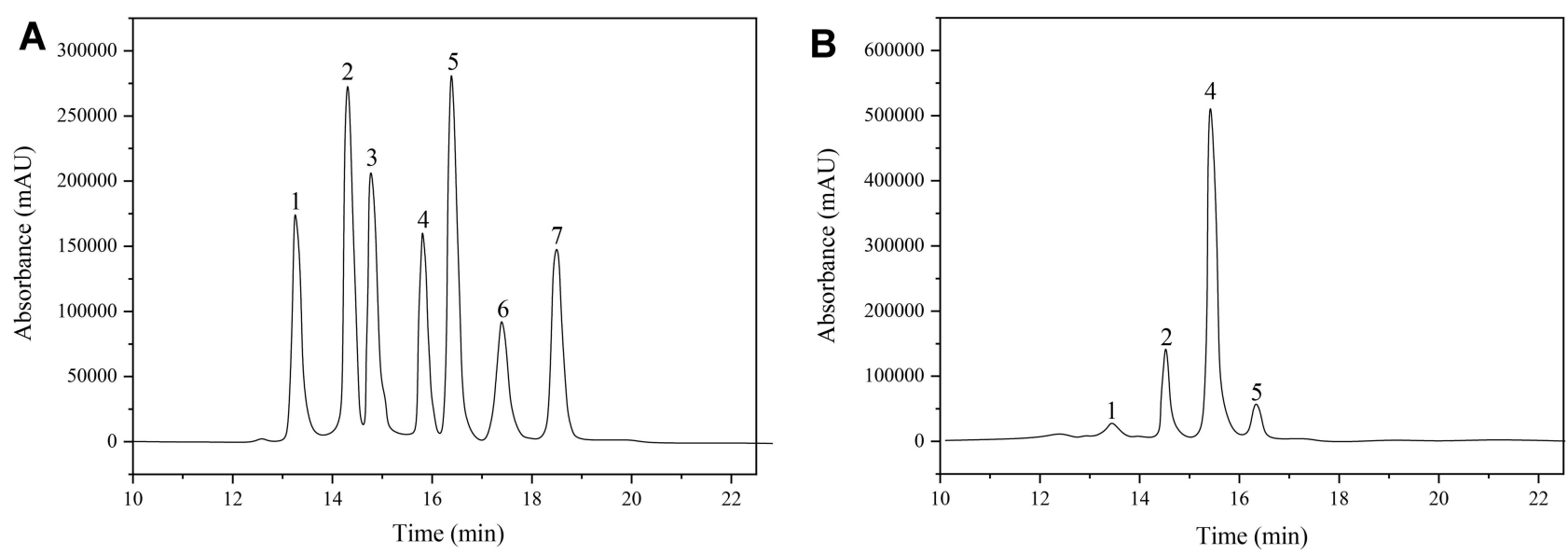

Figure 4 HPLC chromatograms. (A) HPLC chromatograms of PMP derivatization of standard monosaccharides; (B) HPLC chromatograms of PMP derivatization of ARPS. Notes: I. Mannose, 2. Ribose, 3. Rhamnose, 4. Glucose, 5. Arabinose, 6. Xylose, 7. Galacturonic acid.

ARPS consisted of glucose, ribose, arabinose, and mannose at a molar ratio of 54.24:13.20:1.09:1.00. In accordance with the HPLC method in the previous study, the purity of ARPS was determined to reach $96.93 \%$.

\section{In-vivo Hepatoprotective Effects of ARPS Drug Concentration Value of Pre-Experimental Results}

CCK-8 was used to detect cell proliferation, and the absorbance value (OD) was measured at $450 \mathrm{~nm}$ to determine the number of viable cells. The greater the OD value is, the stronger the cell activity. As shown in Table 1 and Figure 5, the OD value of CCK-8 cell in rats with $\mathrm{CCl}_{4}$ induced liver injury in different dose groups was statistically analysed. The OD of the HD group was significantly higher than that of the MD group and the LD group. The OD value of the HD group increased gradually with the prolongation of blood withdrawal time $(0,4,8,12$, and 24 h), the OD value of the HD group gradually increased, reached the maximum at $24 \mathrm{~h}$, and then slightly decreased at $48 \mathrm{~h}$. This trend was compared with that of the MD and LD groups at different time points. According to the above cell growth curve and statistical results, the OD value of HD group was higher than that of the MD and LD groups at $24 \mathrm{~h}$ blood collection time point, indicating that the cell activity was the highest at this time point. Therefore, HD group $(400 \mathrm{mg} / \mathrm{kg} / \mathrm{d})$ was selected for drug intervention in liver injury model induced by $\mathrm{CCl}_{4}$. Blood samples were collected at $24 \mathrm{~h}$ after ARPS intervention for the detection of various indicators.

\section{Effects of ARPS on the Activity of Serous ALT and AST}

As illustrated in Table 2, the activities of serous ALT and AST in the MC group significantly increased $(P<0.05)$ compared with those in the NC group. Intervention by either lentinan or ARPS significantly decreased $(P<$ $0.05)$ the activities of ALT and AST compared with the

Table I The OD Value of CCK-8 Cell in Rats with $\mathrm{CCl}_{4}$-Induced Liver Injury in Different Dose Groups $(n=3, \bar{X} \pm s)$

\begin{tabular}{|c|c|c|c|c|c|}
\hline $\begin{array}{l}\text { Blood } \\
\text { Collection } \\
\text { Time Point }\end{array}$ & $\begin{array}{l}\text { Low-Dose Group of ARPS } \\
\text { (100 mg/kg/d, LD Group) }\end{array}$ & $\begin{array}{l}\text { Medium-Dose Group of ARPS } \\
\text { (200 mg/kg/d, MD Group) }\end{array}$ & $\begin{array}{l}\text { High-Dose Group of ARPS } \\
(400 \mathrm{mg} / \mathrm{kg} / \mathrm{d}, \text { HD Group) }\end{array}$ & $F$ value & $P$ value \\
\hline $\mathrm{Oh}$ & $0.760 \pm 0.031$ & $0.808 \pm 0.025$ & $0.785 \pm 0.052$ & 2.404 & 0.171 \\
\hline $4 \mathrm{~h}$ & $0.792 \pm 0.062$ & $0.777 \pm 0.008$ & $0.839 \pm 0.044$ & 1.621 & 0.274 \\
\hline $8 \mathrm{~h}$ & $0.766 \pm 0.014$ & $0.816 \pm 0.038$ & $0.852 \pm 0.052^{\mathrm{a}}$ & 3.832 & 0.085 \\
\hline $12 \mathrm{~h}$ & $0.783 \pm 0.046$ & $0.80 \mathrm{I} \pm 0.068$ & $0.874 \pm 0.027$ & 2.750 & 0.142 \\
\hline $24 \mathrm{~h}$ & $0.774 \pm 0.023$ & $0.798 \pm 0.033$ & $0.890 \pm 0.012^{\mathrm{a}, \mathrm{b}}$ & 19.454 & 0.002 \\
\hline $48 \mathrm{~h}$ & $0.778 \pm 0.030$ & $0.784 \pm 0.038$ & $0.834 \pm 0.024$ & 3.010 & 0.124 \\
\hline$F$ value & 0.188 & 0.398 & 1.370 & & \\
\hline$P$ value & 0.939 & 0.806 & 0.312 & & \\
\hline
\end{tabular}

Notes: ${ }^{\text {a}} \mathrm{HD}$ group compared with LD group, $P<0.05$; ${ }^{\text {} H D}$ group compared with MD control group, $P<0.05$. 


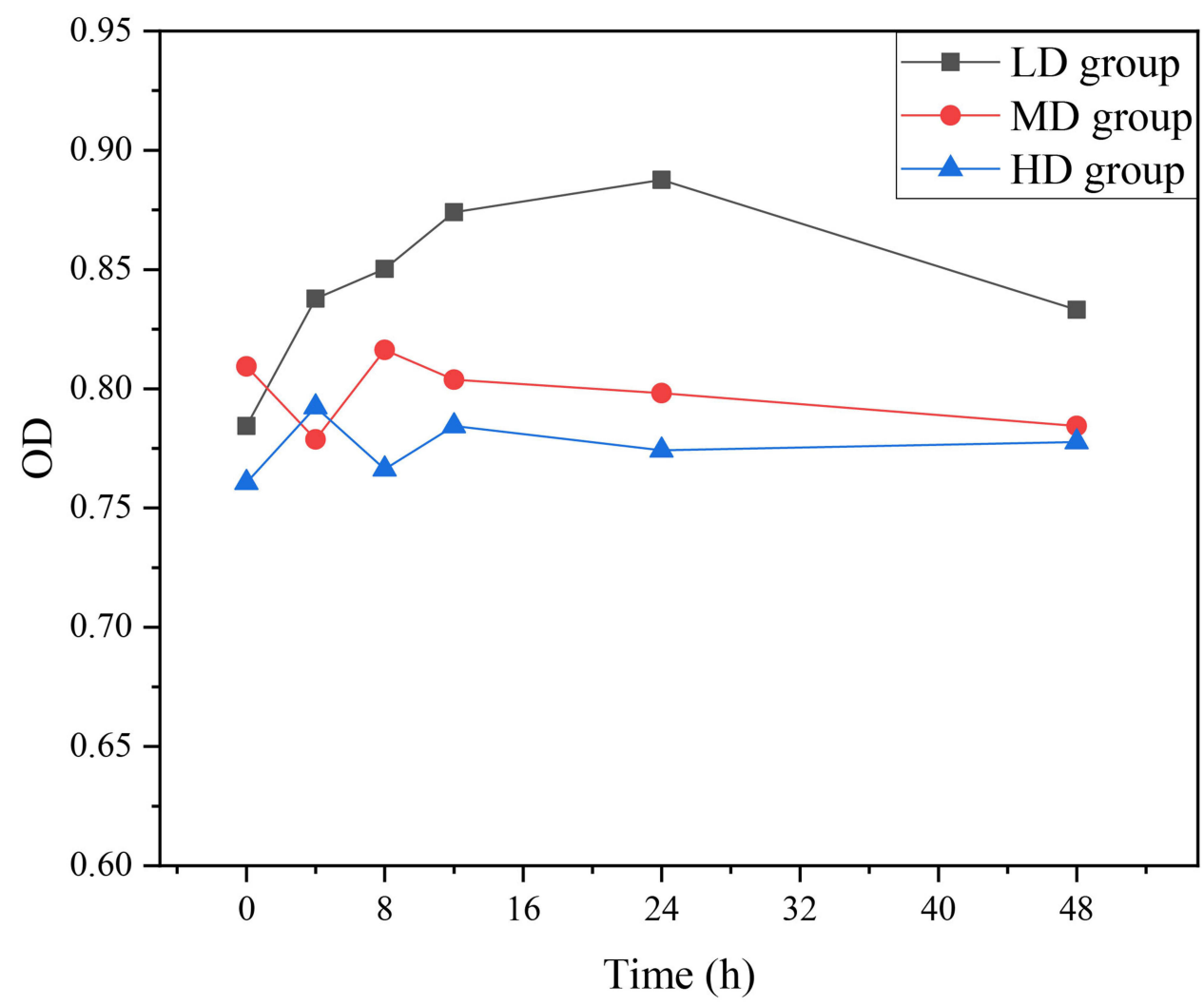

Figure 5 The growth curve of CCK-8 cell in rats with $\mathrm{CCl}_{4}$-induced liver injury in different dose groups.

MC group. ARPS administration reduced the activities of ALT and AST 51.16\% and 54.60\%, respectively, while lentinan, a standard drug, decreased these activities by $64.76 \%$ and $54.78 \%$, respectively.

\section{Effects of ARPS on the Content of Serous MDA, GSH and NOS}

As described in Table 3, the contents of serous MDA and NOS in rats with $\mathrm{CCl}_{4}$-induced liver injury in the $\mathrm{MC}$ group significantly increased $(P<0.05)$ compared with those in the NC group. However, intervention by either lentinan or ARPS could be reversed in the opposite direction. Compared with

Table 2 The Activity of ALT and AST in Rats with $\mathrm{CCl}_{4}$-Induced Liver Injury in Different Groups $(n=5, \bar{X} \pm s)$

\begin{tabular}{|l|l|l|}
\hline Group & ALT (U/L) & AST (U/L) \\
\hline Normal control group & $31.87 \pm 6.65$ & $42.74 \pm 5.98$ \\
Model control group & $113.40 \pm 13.45^{\mathrm{a}}$ & $159.22 \pm 15.53^{\mathrm{a}}$ \\
Positive control group & $48.64 \pm 9.91^{\mathrm{a}, \mathrm{b}}$ & $71.99 \pm 12.16^{\mathrm{a}, \mathrm{b}}$ \\
ARPS group & $55.38 \pm 16.10^{\mathrm{a}, \mathrm{b}}$ & $72.28 \pm 5.77^{\mathrm{a}, \mathrm{b}}$ \\
F value & 43.177 & 110.818 \\
$P$ value & 0.000 & 0.000 \\
\hline
\end{tabular}

Notes: ${ }^{a}$ Experimental group compared with normal control group, $P<0.05$

${ }^{b}$ Experimental group compared with model control group, $P<0.05$. the NC group, the MC group showed sharply reduced hepatic GSH levels $(P<0.05)$ after intragastric administration of $\mathrm{CCl}_{4}$. However, the contents of serous GSH in the ARPS group considerably increased compared with those in the MC group. By contrast, ARPS administration reduced the contents of MDA and NOS by $41.62 \%$ and $46.99 \%$, respectively. These values were close to those obtained with the standard drug lentinan (43.97\% and 54.81\%, respectively). Moreover,

Table 3 The Content of MDA, GSH, and NOS in Rats with $\mathrm{CCl}_{4}$ -Induced Liver Injury in Different Groups ( $n=5, \bar{X} \pm s)$

\begin{tabular}{|l|c|c|c|}
\hline Group & $\begin{array}{c}\text { MDA } \\
(\mathbf{n m o l} / \mathbf{m L})\end{array}$ & $\begin{array}{c}\text { GSH } \\
(\mathbf{m g} \text { GSH/L) }\end{array}$ & NOS (U/mL) \\
\hline $\begin{array}{l}\text { Normal control } \\
\text { group }\end{array}$ & $4.85 \pm 0.22$ & $3.59 \pm 0.69$ & $3.83 \pm 1.61$ \\
$\begin{array}{l}\text { Model control } \\
\text { group }\end{array}$ & $10.62 \pm 0.53^{\mathrm{a}}$ & $0.53 \pm 0.26^{\mathrm{a}}$ & $35.32 \pm 10.02^{\mathrm{a}}$ \\
$\begin{array}{l}\text { Positive control } \\
\text { group }\end{array}$ & $5.95 \pm 0.78^{\mathrm{a}, \mathrm{b}}$ & $2.00 \pm 0.89^{\mathrm{a}, \mathrm{b}}$ & $15.96 \pm 4.05^{\mathrm{a}, \mathrm{b}}$ \\
$\begin{array}{l}\text { ARPS group } \\
\text { F value }\end{array}$ & $6.20 \pm 0.69^{\mathrm{a}, \mathrm{b}}$ & $1.93 \pm 0.52^{\mathrm{a}, \mathrm{b}}$ & $18.72 \pm 10.02^{\mathrm{a}, \mathrm{b}}$ \\
$P$ value & 69.757 & 19.336 & 69.757 \\
& 0.000 & 0.000 & 0.000 \\
\hline
\end{tabular}

Notes: ${ }^{\text {a}}$ Experimental group compared with normal control group, $P<0.05$; ${ }^{\mathrm{b}}$ Experimental group compared with model control group, $P<0.05$. 
compared with the MC group, the ARPS group showed increased hepatic GSH levels of $264.15 \%$, which was quite comparable to the levels from lentinan treatment (277.36\%).

\section{Histopathological Results}

As shown in Figure 6, the rats in the NC group had a normal liver lobular architecture and cell structure, the liver plate was neatly arranged, and no inflammatory infiltration was found (Figure 6A). On the contrary, extensive areas of necrosis with loss of hepatocytes architecture around the blood vessels, a change in hepatic cell cytoplasmic acid, and dissoluted and necrotized partial hepatic cells with a small amount of inflammation were obviously observed in the livers of rats in the MC group (Figure 6B). However, intervention by either lentinan or ARPS could reduce the injured area, edematous liver cells, and $\mathrm{CCl}_{4^{-}}$ induced inflammatory infiltration (Figure 6C and D).

\section{Discussion}

In this experiment, the ultrasonic-assisted water extraction method was adopted in accordance with the polarity of the polysaccharide. The extracted crude polysaccharides were removed from the protein by Sevage method and purified by HSCCC. HSCCC has been widely applied in the separation and purification of polysaccharides. ${ }^{35,39}$ Selecting a suitable diphase solvent system is a crucial step of HSCCC. ${ }^{40}$ Systematic search for an appropriate two-phase solvent system depends on the suitable $K$, which is the ratio of solute distributed between the mutually balanceable two solvent phases and satisfactory retention of the stationary phase in the column. The proper $K$ values to the analyte for HSCCC are $0.5<K<1.0 .^{36}$ The effect of the mass fraction of phosphate in the range of $12-26 \%$ on $K$ was investigated. When the amount of phosphate $\geq 16 \%$, the two phases began to separate. Increasing the amount of salt could make the two phases separate and make partially dissolved ARPS change from the lower phase to the upper phase. According to the "similar miscibility" principle, ARPS is excessively enriched in the polar phosphate phase (mobile phase), which is not conducive to the separation of ARPS in HSCCC. Increasing the amount of phosphate could change the phosphate aqueous solution from an unsaturated state to a saturated state. When the hydrophobicity and ionic strength of the solution have changed, the polysaccharides could not be combined with water molecules in the phosphate phase, and parts of polysaccharides are dissolved in the polymer phase (stationary phase), which increases $K$. The optimum mass fraction of $\mathrm{K}_{2} \mathrm{HPO}_{4}-\mathrm{KH}_{2} \mathrm{PO}_{4}(1: 1)$ is $20 \%$. This experiment adopted PEG $1000-\mathrm{K}_{2} \mathrm{HPO}_{4}-\mathrm{KH}_{2}$ $\mathrm{PO}_{4}-\mathrm{H}_{2} \mathrm{O}(1.25: 1: 1: 6.75$, w/w) as the solvent system with ARPS purified by HSCCC, which obtained $K=0.868$. $\mathrm{V}_{\mathrm{u}}$ $: \mathrm{V}_{\mathrm{L}}=35: 47$, the layered time was $2.1 \mathrm{~min}$, and the retention rate of the stationary phase was $32.59 \%$.
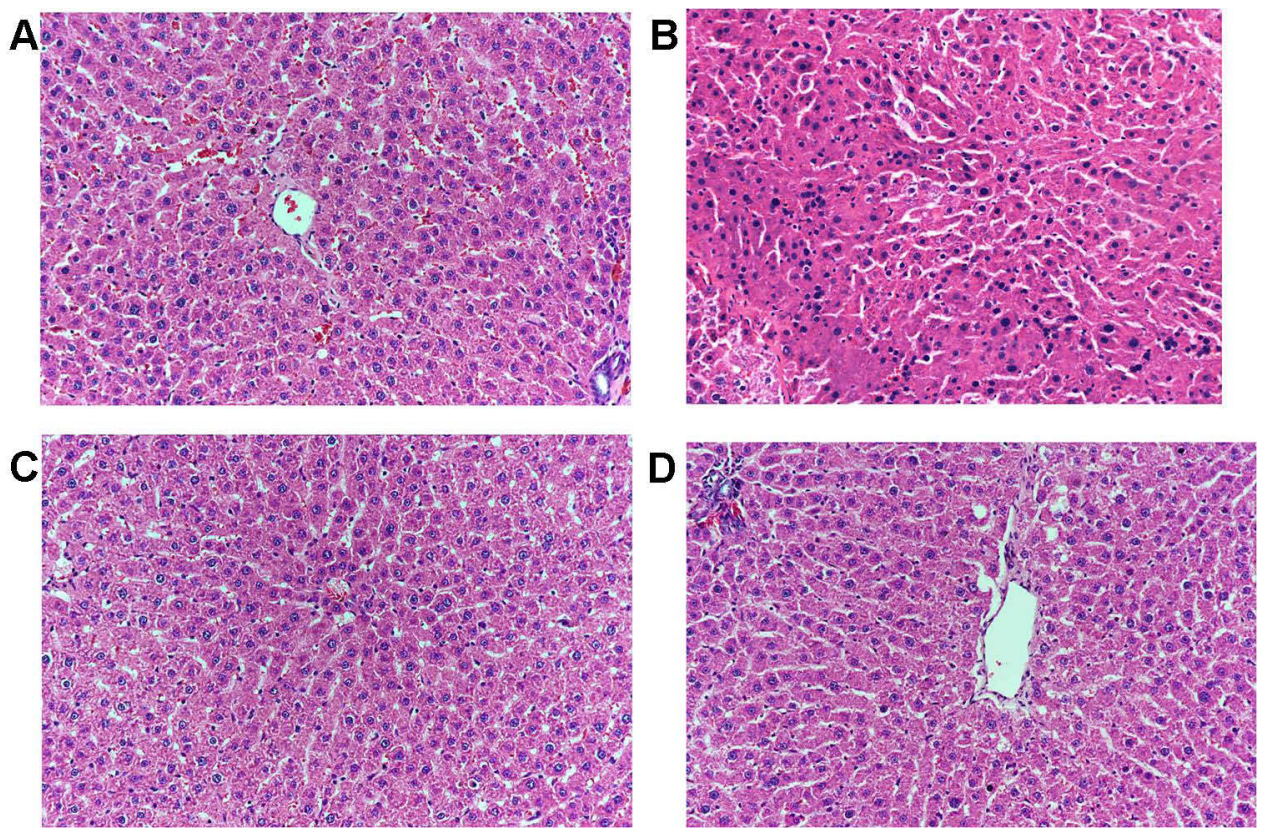

Figure 6 Histopathological changes in rat livers, stained with H\&E (200x). (A) The liver slices of normal rats without damage; (B) with CCl 4 damaged group; (C) treated with lentinan as positive control group; (D) treated with ARPS group. 
The purity of lentinan was identified via gel chromatography, whose operation processes were tedious and time consuming. ${ }^{41}$ In reference to relevant literature, ${ }^{42}$ the purity of ARPS was analyzed by HPGPC in the present study. Oneness and symmetry analysis of the chromatograms preliminarily verified that ARPS was a single component with high purity. The average molecular weight of ARPS was $28,518 \mathrm{Da}$, as measured by HPGPC. Different plant polysaccharides have different molecular weights. For example, the molecular weight of Glycyrrhiza polysaccharide was $5000,{ }^{43}$ while the relative molecular weight of tremella polysaccharide was $1.26 \times 10^{6}-1.39 \times 10^{6}$. ${ }^{44}$ Polysaccharides with different molecular weights have different biological activities. Thus, the molecular weight of polysaccharides is obtained via HPGPC in this study.

In the present study, the monosaccharide composition of ARPS hydrolysis product was determined via HPLC, and five kinds of monosaccharide, including mannoses, ribose, glucoses, arabinoses and glucoses, were successfully separated. The composition of polysaccharide with wild $A$. roxburghii was composed of fucose: mannose: galactose: arabinose: rhamnose $=1.7: 1.3: 1.2: 1.1: 1.0$ and the mole ratio of difference was bigger, ${ }^{45}$ indicating that artificially planted wild $A$. roxburghii has a different monosaccharide composition.

$\mathrm{CCl}_{4}$ is a common hepatotoxic and classic exogenous compound that could induce a chemical liver injury model. ${ }^{46}$ $\mathrm{CCl}_{4}$-induced liver injury animal models are widely used in various liver drug screenings. Pharmacological studies have found that many Chinese medicines have an enhanced therapeutic effect on liver disease. ${ }^{47}$ In the present study, the activities of serum ALT and AST were significantly increased $(P<0.05)$ after $\mathrm{CCl}_{4}$ intervention, thus suggesting $\mathrm{CCl}_{4}$ induced hepatotoxicity. However, the activities of serum ALT and AST dramatically declined $(P<0.05)$ in rats who underwent ARPS treatment, indicating that ARPS relieved $\mathrm{CCl}_{4}$ induced liver injury. Oxidative stress has been considered as a major mechanism of $\mathrm{CCl}_{4}$ toxicity. This study suggested that oxidative stress markers MDA and NOS increased and the antioxidant enzyme GSH decreased after $\mathrm{CCl}_{4}$ injection, indicating that oxidative stress was caused by inflammatory response factors and inflammatory mediators in the injured liver induced by $\mathrm{CCl}_{4}$ in rats. In rats intervened with ARPS, these effects were attenuated, thereby suggesting that the hepatoprotective effects of ARPS were, at least in part, caused by their radical scavenging and antioxidant activities. However, the exact molecular mechanisms and signaling pathways remain to be further clarified. The pathological section of the rat liver tissue after H\&E staining showed that the liver plate of the MC group was slightly disordered. The liver cells were obviously edematous, with some showing balloon-like changes, and the liver tissue around the central vein had obvious edema and fat-like changes, which may be caused by the metabolism of $\mathrm{CCl}_{4}$ first passing through the central vein and then entering the liver tissue. In the ARPS group, the liver plates of the rats returned to a regular arrangement, the hepatocyte degeneration and edema improved, and no inflammatory cell infiltration was found. These findings were comparable to those in the PC group and tended to be normal. The results of histopathological morphology and various liver function indicators were consistent, suggesting that ARPS could reduce $\mathrm{CCl}_{4}$-induced liver injury and enhance the regeneration ability of liver cells.

\section{Conclusion}

This study provided a detailed description of the concrete procedure and essential prerequisite for purification of polysaccharide from A. raxburghii by HSCCC. The results confirmed that ARPS was a water-soluble polysaccharide mainly composed of glucose, ribose, arabinose, and mannose at a molar ratio of 54.24:13.20:1.09:1.00 and molecular mass of 28,518 Da. Meanwhile, ARPS showed hepatoprotective effects on rat liver injury induced by $\mathrm{CCl}_{4}$. The underlying mechanisms of these effects may be connected to the attenuation of oxidative stress. One limitation of this study is that it did not delve into the spatial conformation of sugar chains. Therefore, further studies are needed to detect the concrete structure of ARPS. Despite its preliminary characterization, this study clearly provided a useful foundation for further pharmacological and mechanical research on ARPS.

\section{Abbreviations}

A. roxburghii, Anoectochilus roxburghii(wall.) Lindl.; ARPS, polysaccharide of Anoectochilus roxburghii (wall.) Lindl.; HSCCC, high-speed counter current chromatography; $\mathrm{CCl}_{4}$, carbon tetrachloride; ALT, alanine amino transferase; AST, aspartate amino transferase; GSH, glutathione; NOS, nitric oxide synthesis; MDA, malondialdehyde; CARPS, crude polysaccharide of A. roxburghii; Mw, molecular weight; PMP, 1-phenyl3-methyl-5-pyrazolone; BW, body weights; NC group, normal control group; MC group, model control group; LD group, low-dose group of ARPS; MD group, medium-dose group of ARPS; HD group, high-dose group of ARPS; CCK-8, Cell Counting Kit-8; PC group, positive control group; ARPS group, ARPS treatment group; 
H\&E, hematoxylin and eosin; HPGP, high-performance gel -permeation chromatography.

\section{Ethics Approval and Consent to Participate}

The ethical approval of this study was provided by the Institutional Ethical Committee of the Faculty of Medicine, Mengchao Hepatobiliary Hospital of Fujian Medical University, Fujian, China (No. 2020_051_01).

\section{Acknowledgments}

The authors wish to express their gratitude to Bin Wang (Department of Pathology, Mengchao Hepatobiliary Hospital of Fujian Medical University, China), Juxiang Zeng (Department of Infectious Diseases, Mengchao Hepatobiliary Hospital of Fujian Medical University, China), and Wenqian Jiang (Department of Infectious Diseases, Mengchao Hepatobiliary Hospital of Fujian Medical University, China) for the support on the manuscript.

\section{Author Contributions}

All authors contributed to data analysis, drafting or revising the article, have agreed on the journal to which the article will be submitted, gave final approval for the version to be published, and agree to be accountable for all aspects of the work.

\section{Funding}

This study was sponsored by the Social Development Project of Fuzhou Science and Technology Bureau, P. R. C. [Grant numbers 2019-SZ-42]; the Special Project Scientific Research of the Fujian Provincial Department of Finance, China [Grant numbers 2019 B029]; and the Natural Fund Project of Fujian Province, China [Grant numbers 2020J011155, 2020J01623).

\section{Disclosure}

The authors report no conflicts of interest in this work.

\section{References}

1. Du XM, Irino N, Furusho N, Hayashi J, Shoyama Y. Pharmacologically active compounds in the anoectochilus and goodyera species. J Nat Med. 2008;62(2):132-148. doi:10.1007/s11418007-0169-0

2. Liu SS, Chen J, Li SC, Zeng X, Meng ZX, Guo SX. Comparative transcriptome analysis of genes involved in GA-GID1-DELLA regulatory module in symbiotic and asymbiotic seed germination of Anoectochilus roxburghii (Wall.) Lindl. (Orchidaceae). Int J Mol Sci. 2015;16(12):30190-30203. doi:10.3390/ijms161226224
3. Magassouba FB, Diallo A, Kouyate M, et al. Ethnobotanical survey and antibacterial activity of some plants used in Guinean traditional medicine. J Ethnopharmacol. 2007;114(1):44-53. doi:10.1016/j. jep.2007.07.009

4. Huang L, Cao Y, Xu H, Chen G. Separation and purification of ergosterol and stigmasterol in Anoectochilus roxburghii (wall) Lindl by high-speed counter-current chromatography. J Sep Sci. 2015;34 (4):385-392. doi: $10.1002 /$ jssc. 201000577

5. Zhang X, Jin M, Liu Y, et al. Oral bioavailability of kinsenoside in beagle dogs measured by LC-MS/MS: improvement of ex vivo stability of a lactone-containing compound. Pharmaceutics. 2018;10 (3):87. doi:10.3390/pharmaceutics 10030087

6. Zeng B, Su M, Chen Q, Chang Q, Wang W, Li H. Protective effect of a polysaccharide from Anoectochilus roxburghii against carbon tetrachloride-induced acute liver injury in mice. $J$ Ethnopharmacol. 2017;200:124-135. doi:10.1016/j.jep.2017.02.018

7. Zeng B, Su M, Chen Q, Chang Q, Wang W, Li H. Antioxidant and hepatoprotective activities of polysaccharides from Anoectochilus roxburghii. Carbohydr Polym. 2016;153:391-398. doi:10.1016/j. carbpol.2016.07.067

8. Yang Z, Zhang X, Yang L, et al. Protective effect of Anoectochilus roxburghii polysaccharide against CCl4-induced oxidative liver damage in mice. Int $J$ Biol Macromol. 2017;96:442-450. doi:10.1016/j.ijbiomac.2016.12.039

9. Qi CX, Zhou Q, Yuan Z, et al. Kinsenoside: a promising bioactive compound from anoectochilus species. Curr Med Sci. 2018;38 (1):11-18. doi:10.1007/s11596-018-1841-1

10. Ye S, Shao Q, Zhang A. Anoectochilus roxburghii: a review of its phytochemistry, pharmacology, and clinical applications. J Ethnopharmacol. 2017;209:184-202. doi:10.1016/j.jep.2017.07. 032

11. Guo Y, Ye Q, Yang S, et al. Therapeutic effects of polysaccharides from Anoectochilus roxburghii on type II collagen-induced arthritis in rats. Int $J$ Biol Macromol. 2019;122:882-892. doi:10.1016/j. ijbiomac.2018.11.015

12. Ikeuchi M, Yamaguchi K, Nishimura T, Yazawa K. Effects of Anoectochilus formosanus on endurance capacity in mice. J Nutr Sci Vitaminol (Tokyo). 2005;51(1):40-44. doi:10.3177/ jnsv. 51.40

13. Masuda K, Ikeuchi M, Koyama T, et al. Suppressive effects of Anoectochilus formosanus extract on osteoclast formation in vitro and bone resorption in vivo. J Bone Miner Metab. 2008;26 (2):123-129. doi:10.1007/s00774-007-0810-8

14. Hsiao HB, Lin H, Wu JB, Lin WC. Kinsenoside prevents ovariectomy-induced bone loss and suppresses osteoclastogenesis by regulating classical NF-kappaB pathways. Osteoporos Int. 2013;24(5):1663-1676. doi:10.1007/s00198-012-2199-Z

15. Liu ZL, Zhang JG, Liu Q, Yi LT, Li YM, Li Y. The vascular protective effects of Anoectochilus roxburghii polysaccharose under high glucose conditions. J Ethnopharmacol. 2017;202:192-199. doi:10.1016/j.jep.2017.03.012

16. Thong NM, Vo QV, Huyen TL, Bay MV, Tuan D, Nam PC. Theoretical study for exploring the diglycoside substituent effect on the antioxidative capability of isorhamnetin extracted from Anoectochilus roxburghii. ACS Omega. 2019;4(12):14996-15003. doi:10.1021/acsomega.9b01780

17. Xu M, Shao Q, Ye S, et al. Simultaneous extraction and identification of phenolic compounds in Anoectochilus roxburghii using microwave-assisted extraction combined with UPLC-Q-TOF-MS /MS and their antioxidant activities. Front Plant Sci. 2017;8:1474. doi:10.3389/fpls.2017.01474

18. Yang LC, Hsieh CC, Lu TJ, Lin WC. Structurally characterized arabinogalactan from Anoectochilus formosanus as an immuno-modulator against CT26 colon cancer in BALB/c mice. Phytomedicine. 2014;21(5):647-655. doi:10.1016/j.phymed.2013. 10.032 
19. Yu X, Lin S, Zhang J, Huang L, Yao H, Li S. Purification of polysaccharide from artificially cultivated Anoectochilus roxburghii (wall.) Lindl. by high-speed counter current chromatography and its antitumor activity. J Sep Sci. 2017;40(22):4338-4346. doi:10.1002/ jssc. 201700340

20. Hua ZT, Ying HL, Yong W. Extraction and determination of the alkaloids in anoectochilus formosanus. Chem Res. 2006.

21. Chen X, Wu Y, Huang L, et al. Magnetic dispersive solid-phase micro-extraction combined with high-performance liquid chromatography for determining nucleotides in Anoectochilus roxburghii (Wall.) Lindl. J Pharm Biomed Anal. 2019;174:432-440. doi:10.1016/j.jpba.2019.06.010

22. Huang L, Zhong T, Chen T, Ye Z, Chen G. Identification of beta-sitosterol, stigmasterol and ergosterin in A. roxburghii using supercritical fluid extraction followed by liquid chromatography/ atmospheric pressure chemical ionization ion trap mass spectrometry. Rapid Commun Mass Spectrom. 2007;21 (18):3024-3032. doi:10.1002/rcm.3181

23. Huang L, Chen T, Ye Z, Chen G. Use of liquid chromatography-atmospheric pressure chemical ionization-ion trap mass spectrometry for identification of oleanolic acid and ursolic acid in Anoectochilus roxburghii (wall.) Lindl. J Mass Spectrom. 2007;42(7):910-917. doi:10.1002/jms.1228

24. Huang L, Cao Y, Chen G. Purification of quercetin in Anoectochilu roxburghii (wall) Lindl using UMAE by high-speed counter-current chromatography and subsequent structure identification. Sep Purif Technol. 2008;64(1):101-107. doi:10.1016/j.seppur.2008.07.021

25. Huang L, Cao Y, Xu H, Chen G. Separation and purification of ergosterol and stigmasterol in Anoectochilus roxburghii (wall) Lindl by high-speed counter-current chromatography. J Sep Sci. 2011;34 (4):385-392. doi:10.1002/jssc.201000577

26. Hui-Fang C, Pin-Pin W, Li-Ying H, et al. Analysis of gastrodine and 4-hydroxybenzyl alcohol in A. roxburghii by HPLC-DAD. Anal Test Technol Instrum. 2015.

27. Lin L, Huang L, Zheng Y. Extraction in polysaccharide of Anoectochilus roxburghii and research of its anti-oxidative. J Fujian Trad Chin Med Coll. 2006;16:37-40.

28. Zhong T, Huang L. Comparison of tumor proteomics of aqueous extraction in Anoectochilus roxburghii. Chin J Hosp Pharm. 2011;31:623-626.

29. Shih CC, Wu YW, Hsieh CC, Lin WC. Effect of Anoectochilus formosanus on fibrosis and regeneration of the liver in rats. Clin Exp Pharmacol Physiol. 2004;31(9):620-625. doi:10.1111/j.14401681.2004.04062.x

30. Wu JB, Chuang HR, Yang LC, Lin WC. A standardized aqueous extract of Anoectochilus formosanus ameliorated thioacetamide-induced liver fibrosis in mice: the role of Kupffer cells. Biosci Biotechnol Biochem. 2010;74(4):781-787. doi:10.1271/bbb.90824

31. Ito Y. Golden rules and pitfalls in selecting optimum conditions for high-speed counter-current chromatography. $J$ Chromatogr A. 2005;1065(2):145-168. doi:10.1016/j.chroma.2004.12.044

32. Li C, Cai J, Geng J, Li Y, Wang Z, Li R. Purification, characterization and anticancer activity of a polysaccharide from Panax ginseng. Int J Biol Macromol. 2012;51(5):968-973. doi:10.1016/j.ijbiomac.2012.06.031

Drug Design, Development and Therapy

\section{Publish your work in this journal}

Drug Design, Development and Therapy is an international, peerreviewed open-access journal that spans the spectrum of drug design and development through to clinical applications. Clinical outcomes, patient safety, and programs for the development and effective, safe, and sustained use of medicines are a feature of the journal, which has also
33. Alsop RM, Vlachogiannis GJ. Determination of the molecular weight of clinical dextran by gel permeation chromatography on TSK PW type columns. J Chromatogr A. 1982;246(2):227-240. doi:10.1016/ s0021-9673(00)95862-x

34. Xavier L, Freire MS, Vidal-Tato I, González-álvarez J. Recovery of phenolic compounds from eucalyptus globulus wood wastes using PEG/phosphate aqueous two-phase systems. Waste Biomass Valori. 2016;8(2):443-452. doi:10.1007/s12649-016-9579-0

35. Yang W, Wang Y, Li X, Yu P. Purification and structural characterization of Chinese yam polysaccharide and its activities. Carbohydr Polym. 2015;117:1021-1027. doi:10.1016/j.carbpol.2014.09.082

36. Pang KS, Cherry WF, Ulm EH. Disposition of enalapril in the perfused rat intestine-liver preparation: absorption, metabolism and first-pass effect. $J$ Pharmacol Exp Ther. 1985;233(3):788-795.

37. Jiang Y, Qi X, Gao K, et al. Relationship between molecular weight, monosaccharide composition and immunobiologic activity of Astragalus polysaccharides. Glycoconj J. 2016;33(5):755-761. doi:10.1007/s10719-016-9669-Z

38. Gao J, Zhang T, Jin ZY, et al. Structural characterisation, physicochemical properties and antioxidant activity of polysaccharide from Lilium lancifolium Thunb. Food Chem. 2015;169:430-438. doi:10.1016/j.foodchem.2014.08.016

39. Wu X, Li R, Zhao Y, Liu Y. Separation of polysaccharides from Spirulina platensis by HSCCC with ethanol-ammonium sulfate ATPS and their antioxidant activities. Carbohydr Polym. 2017;173:46 5-472. doi:10.1016/j.carbpol.2017.06.023

40. Khan BM, Liu Y. High speed counter current chromatography: overview of solvent-system and elution-mode. J Liq Chromatogr Relat Technol. 2018;41(10):629-636. doi:10.1080/10826076.2018.1499528

41. Ren G, Xu L, Lu T, Yin J. Structural characterization and antiviral activity of lentinan from Lentinus edodes mycelia against infectious hematopoietic necrosis virus. Int $J$ Biol Macromol. 2018;115:1202-1210. doi:10.1016/j.ijbiomac.2018.04.132

42. Zhang Z, Guo L, Yan A, Feng L, Wan Y. Fractionation, structure and conformation characterization of polysaccharides from Anoectochilus roxburghii. Carbohydr Polym. 2020;231:115688. doi:10.1016/j. carbpol.2019.115688

43. Zhao Y, Song X, Zhang L, Yu T. High gel chromatography determination of glycyrrhiza polysaccharide molecular weight and molecular weight distribution. J Tianjin Trad Chin Med. 2015;32(1):46-48.

44. Liu Q, Ning J, Ding K. Based on the efficient and gel permeation chromatography tremella polysaccharide quality control study. Chin Herb Med. 2011;42(9):1732-1735.

45. Yang X. The Protective Effect of ARPS on the Alcoholic Liver Injury Mice and ARPS Granule Prepared. Fujian Medical University; 2017.

46. Scholten D, Trebicka J, Liedtke C, Weiskirchen R. The carbon tetrachloride model in mice. Lab Anim. 2015;49(1 Suppl):4-11. doi:10.1177/0023677215571192

47. Yin L, Yu H, Peng J. Molecular mechanism of carbon tetrachloride induced liver damage and the progress of Chinese medicine intervention. J Mod Appl Pharm China. 2015;32(9):1147-1155. been accepted for indexing on PubMed Central. The manuscript management system is completely online and includes a very quick and fair peer-review system, which is all easy to use. Visit http://www. dovepress.com/testimonials.php to read real quotes from published authors. 